\title{
An innovative nasal continuous positive airway pressure assembly
}

\author{
Kapil Chaudhary, Munisha Agarwal ${ }^{1}$
}
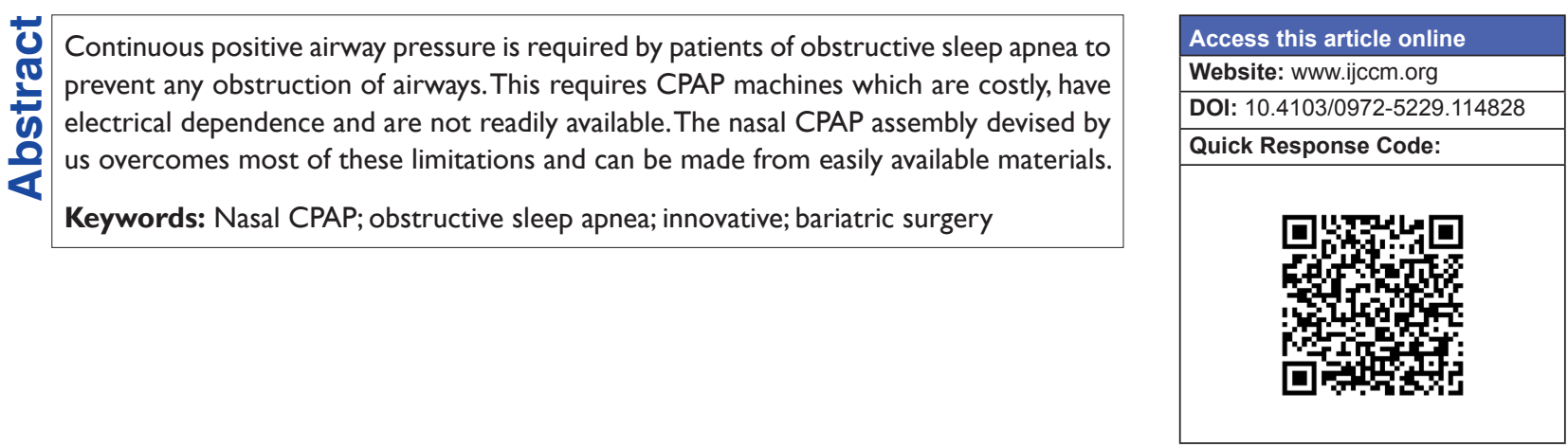

The continuous positive airway pressure (CPAP) machines, used for the treatment of obstructive sleep apnea (OSA), deliver a stream of compressed air (to a nasal pillow, nose mask, or full-face mask) to generate a positive pressure, which splints the airway ${ }^{[1]}$ so that unobstructed breathing becomes possible, thus preventing and/or reducing apnea/hypopnea. The commercially available CPAP machines require proper care and maintenance, use substantial electrical power, are noisy, and need skilled personnel for their operation. The dependence on electrical backup/batteries and non-availability of newer compact machines in every set up are other limiting factors for their use during transportation despite the current availability of a variety of devices. The interfaces used with the CPAP machines add to the problems of improper fit, leak, poor patient tolerance, non-compliance, aerophagia, rhinitis, runny nose, and skin breakdown.$^{[1]}$ Despite the modifications in newer interfaces, the additional need for improvement in interfaces for delivering $\mathrm{CPAP}$ has been emphasized in literature. ${ }^{[1]}$

\section{From:}

Department of Anaesthesiology and Intensive Care, Maulana Azad Medical College and Associated GB Pant Hospital, ${ }^{1}$ Anaesthesiology and Intensive Care, Maulana Azad Medical College and Associated Hospitals, New Delhi, India

Correspondence:

Dr. Kapil Chaudhary, Department of Anaesthesiology and Intensive Care, Maulana Azad Medical College and Associated GB Pant Hospital, New Delhi, India. E-mail: kapsdr@yahoo.com
We share our experience with an assembly we have devised at our center that can be used to deliver CPAP to patients with minimization of most of the above problems. The assembly is formed by interposing a nasal pronged tube assembly (described below) between a chest drain assembly and a humidified air/oxygen source [Figure 1a]. The portion of twin bore nasal oxygen set (OXY SET, Romsons Sci. and Surg. Ind. (P) Ltd., Agra, India) bearing nasal prongs is cut on either side, and the horizontal tubing of the cut portion containing prongs is then transversely split open. [Figure 1b]. Two holes corresponding to the size of nasal prongs are created in a cut portion (about 4-5 cm in length) of an $8.5 \mathrm{~mm}$ ID endotracheal tube. The cut prongs are then inserted into this tube and prongs taken out through the holes to the exterior, using a blunt forceps, forming the nasal pronged tube assembly. This assembly is then interposed between a chest drain assembly at its distal end and humidified air/ oxygen tubing at its proximal end using an appropriate universal connector [Figure 1a]. The desired amount of CPAP is determined by the depth of chest drain tubing in the water seal of the chest drain assembly. This assembly can be easily fitted on to the patient's face using the adjustable tubing of the nasal oxygen set [Figure 1]. The loops of adjustable tubings of the nasal oxygen set are fitted on the connector attached to the oxygen tubing on one side and the chest drain connector on other side [Figures $1 \mathrm{a}$ and $1 \mathrm{c}$ ] and then passed over the ears to 

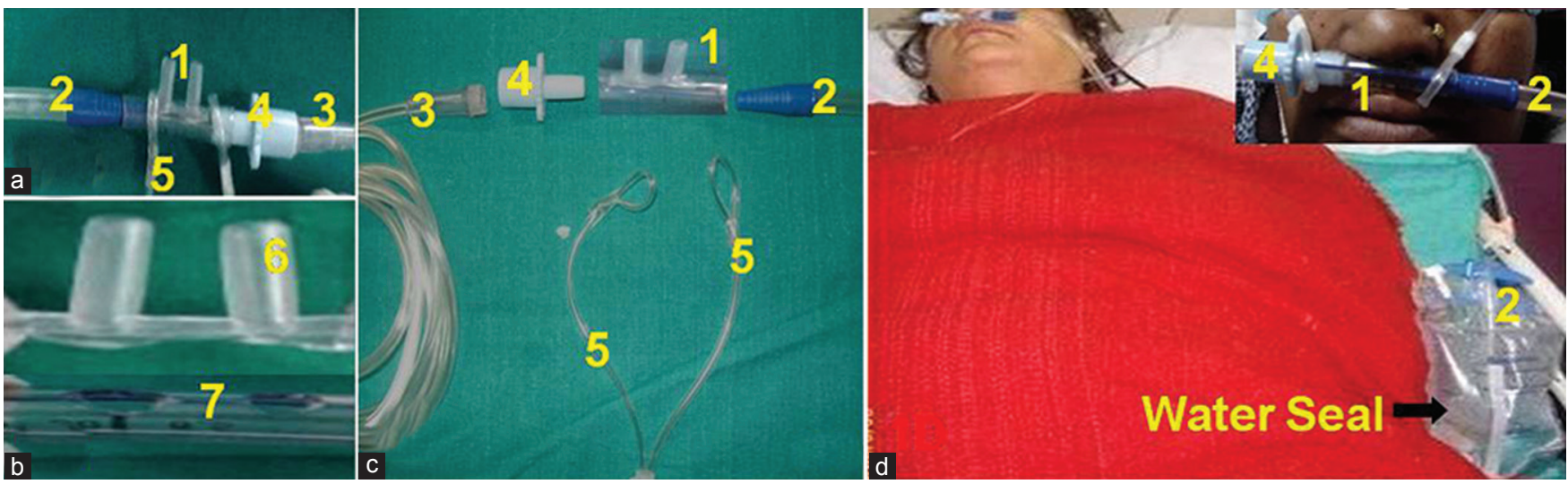

Figure I: The light weight nasal CPAP assembly (a) Formed by interposing nasal pronged tube assembly (I) between water filled chest drain assembly (2) and humidified air/oxygen source (3) Via appropriate universal connector (4). It can be easily fitted on to patient (d) Using the adjustable tubings (5) of nasal oxygen set. (b) The twin prongs of transversely split opened portion of nasal oxygen set, (6) which can be exteriorized through holes made in cut portion of an endotracheal tube (7), to form the nasal pronged tube assembly ( 1 ) This assembly can be easily made by assembling these easily available materials.(c)

support the nasal pronged assembly [Figure 1d]. The water seal is appropriately supported so as to prevent any drag. Initial flows of about 8-10 L/min are needed to pressurize the system, which can thereafter be decreased to $4-8 \mathrm{~L} / \mathrm{min}$ so as to achieve continuous bubbling in water seal and desirable patient parameters.

It is a light-weight assembly and can be devised with the easily available equipments in any operation room (OR) or intensive care unit (ICU) or ward. It also has the advantage of better patient tolerance, being noise- and maintenance-free, and helps in easy CPAP delivery, even during transportation without the need for "clumsy" battery/inverter. When applied and appropriately pressurized, the assembly causes the non-uniform flow of gas bubbles across the water seal (forming an oscillatory pressure delivery system), which results in transmission of mechanical vibrations into the chest.

The assembly faces some limitations similar to other interfaces used for delivering CPAP. There may be a concern of minor leak with this assembly. However, such a leak, which is also seen with other interfaces, ${ }^{[1,2]}$ is acceptable as long as the ventilation and oxygenation are not hindered ${ }^{[2]}$ The proper working of the assembly can be adjudged by continuous slow, gentle bubbling in the water seal. Absence of bubbling could indicate circuit leak, reduced circuit flow, obstruction from secretions, or large patient air leak, usually from open mouth. The leak may be effectively minimized by checking the assembly throughout its entire length and tightening its connections, chin strapping using a paper tape and use of appropriate-sized prongs. The limitation of disconnection, which is similar to that seen with other such equipment, can also be minimized by ensuring tightening of connections before and during use.
The efficacy of the system was checked by attaching the pressure transducers of anesthesia workstation (Galaxy Meditec m-951-2889, Galaxy Meditech, England) to the chest drain side of the nasal pronged tube assembly through appropriate holes and checking for the pressure generated. The pressure generated was almost equal to the depth of chest drain tubing in the water seal with a variation of $1-2 \mathrm{~cm} \mathrm{H}_{2} 0$ in some cases. Though this assembly does not aim to replace the widely available commercial machines, it may be useful in gaining time and for short-term use as in post-operative room/ward and during transportation when such commercial machines may not be available/feasible.

CPAP using under-water seal has been previously used for one lung ventilation. ${ }^{[3]}$ However, the use of CPAP using water seal device by the technique used by us for post-operative management has largely remained unexplored. We have used this assembly post-operatively in 4 patients undergoing bariatric surgery (BMI varying from $45-57 \mathrm{~kg} / \mathrm{m}^{2}$ ) with Montefiore Obesity Surgery Score 3 and history of moderate to severe OSA admitted in surgical ICU. The assembly resulted in improvement of patient's $\mathrm{SpO}_{2}$ by $4-5 \%$ and was tolerated well. Also, the assembly was very useful in decreasing the work of breathing and maintaining oxygenation post-operatively in 3 patients with perforation peritonitis and history of COPD, who required PEEP intra-operatively. The patients reported better tolerance compared to the conventional CPAP machine, though the improvement in $\mathrm{SpO}_{2}$ was similar with the assembly and conventional CPAP machine when compared to a face mask. The patients gave written consent for publishing the effects of using the assembly. Though we have used it for delivering CPAP for short-term in OR/surgical ICU and while transportation in few patients, it has the potential 
of being used in any patient needing CPAP in medical/ surgical ICU while weaning. The use of pediatric nasal prongs, in place of the adult nasal prongs, may perhaps extend its use in pediatric population.

\section{References}

1. Kakkar RK, Berry RB. Positive Airway Pressure Treatment for Obstructive Sleep Apnea. Chest 2007;132:1057-72.

2. Chang DW. Noninvasive positive pressure ventilation. In:
Chang DW, editor. Clinical Application of Mechanical Ventilation. $3^{\text {rd }}$ ed. New Delhi: Cengage Learning India Private Limited; 2006. p. 197-9.

3. Baraka A, Sibai AN, Nawfal M, Muallem M. Underwater seal for CPAP oxygenation during one-lung ventilation by a Univent tube. Middle East J Anesthesiol 1996;13:581-3.

How to cite this article: Chaudhary K, Agarwal M. An innovative nasal continuous positive airway pressure assembly. Indian J Crit Care Med 2013;17:104-6.

Source of Support: Nil, Conflict of Interest: None declared.

\section{Author Help: Reference checking facility}

The manuscript system (www.journalonweb.com) allows the authors to check and verify the accuracy and style of references. The tool checks the references with PubMed as per a predefined style. Authors are encouraged to use this facility, before submitting articles to the journal.

- The style as well as bibliographic elements should be $100 \%$ accurate, to help get the references verified from the system. Even a single spelling error or addition of issue number/month of publication will lead to an error when verifying the reference.

- Example of a correct style

Sheahan P, O'leary G, Lee G, Fitzgibbon J. Cystic cervical metastases: Incidence and diagnosis using fine needle aspiration biopsy. Otolaryngol Head Neck Surg 2002;127:294-8.

- Only the references from journals indexed in PubMed will be checked.

- $\quad$ Enter each reference in new line, without a serial number.

- Add up to a maximum of 15 references at a time.

- If the reference is correct for its bibliographic elements and punctuations, it will be shown as CORRECT and a link to the correct article in PubMed will be given.

- If any of the bibliographic elements are missing, incorrect or extra (such as issue number), it will be shown as INCORRECT and link to possible articles in PubMed will be given. 